\title{
Effect of vitamin B17 on experimentally induced colon cancer in adult male albino rat
}

\author{
W. Badr El-Kholy, S.A. Abdel-Rahman, F.E.-N. Abd El-Hady El-Safti, N. Mohey Issa \\ Human Anatomy and Embryology, Faculty of Medicine, Menoufia University, Menoufia, Shibin Al-koum, Egypt
}

[Received: 13 December 2019; Accepted: 23 January 2020]

Background: Colon cancer is considered to be the third most common cancer worldwide. At diagnosis of colon cancer, 3.7-11\% developed bone metastasis. Diet based strategies are important for prevention and treatment of colon cancer. This study investigated the effect of vitamin B17 on a DMH induced rat model of colon cancer.

Materials and methods: Eighty young adult male albino rats were divided into five groups: group I (control group), group II (vitamin B17), group III (colon cancer), group IV (protected) and group V (treated). Distal colon sections were prepared for light and scanning electron microscopic examination. Lumbar vertebrae specimens were prepared for light microscopic study. Morphometric and statistical analysis were done.

Results: In comparison with the control, both colon cancer and treated groups showed invasion of the colonic tissue by pleomorphic branching colonic glands of variable shapes and sizes lined with dysplastic elongated hyperchromatic nuclei with frequent mitotic figures or stratified multi-layered crowded nuclei with an extremely significant $(p<0.0001)$ reduction of goblet cell number when compared to the control together with major pathological bone changes were observed in colon cancer and the treated groups.

Conclusions: While the protected group showed impressive improvement of all previously mentioned diameters. (Folia Morphol 2021; 80, 1: 158-169)

Key words: colon cancer rat, vitamin B17

\section{INTRODUCTION}

Colon cancer is often found in patients aged 50 years or older. However, recent interest in Egyptian colon cancer has been raised when epidemiological studies revealed a high incidence of this disease among young Egyptian population aged between 30 and 35 years [14]. The aetiology of colon cancer is multifactorial, including genetic, environmental and dietary factors [2]. At diagnosis of colon cancer, 3.7$-11 \%$ developed bone metastasis [12]. Animal models are good ways to study the disease development. In addition, these models allow for studying the pre- vention of colon cancer [7]. Currently, experimental models use 1,2-dimethylhydrazine (DMH) which is a potent colon carcinogen and the most widely used colon cancer inducer. DMH causes oxidative stress by methylating the molecules of colonic epithelial cells [5]. Colon cancer is considered to be a preventable disease $[17,25]$.

Vitamin B17, commonly known as Laetrile or Amygdalin, is claimed to be a potential natural chemotherapeutic agent found particularly in the seeds of common fruits such as apricots, peaches and apples [9]. The anti-neoplastic effect of vitamin B17 is attribut- 
ed to its ability to induce apoptosis in tumour cells. It is also claimed to reduce the mitotic activity of these cells [18]. It would be therefore worthwhile to experimentally induce a rat model of colon cancer and study the possible role of vitamin B17 whether protective or curative.

\section{MATERIALS AND METHODS}

DMH. A product of Sigma-Aldrich (Munich, Germany), was available in the form of white powder. It was dissolved in $1 \mathrm{mM}$ EDTA-Normal saline. It was given to the rat by intra-peritoneal injection (IP) at a dose of $20 \mathrm{mg} / \mathrm{kg} / \mathrm{body}$ weight once weekly for 5 consecutive weeks [23].

Vitamin B17. It was obtained online from (IHerb, California) in the form of capsules. The container served 100 capsules, each capsule served $100 \mathrm{mg}$ Apricot seed extract (vitamin B17; Amygdalin). It was given at a dose of $300 \mathrm{mg} / \mathrm{kg}$ bodyweight daily by oral tube [24].

Eighty young adult male albino rats, weighing between 160 and $180 \mathrm{~g}$ were obtained from Theodor Bilharz Institute's animal house, El-Warraq, Giza, Egypt. They were fed standard diet. Ethical approval was obtained from the animal house of Theodor Bilharz Institute. Animals were divided into five main groups:

- Group I (control group): this group consisted of 10 male albino rats; they were subdivided into subgroup la: consisted of 5 rats; they were kept without any treatment until the end of the study; subgroup Ib: consisted of 5 rats, they received 1 mM EDTA intra-peritoneal once weekly until the end of the study;

- Group II (vitamin B17 group): consisted of 10 rats, they received the calculated dose of vitamin B17 orally daily for 5 weeks;

- Group III (colon cancer [CC] induced group): consisted of 20 rats, received the calculated dose of DMH IP once weekly for 5 weeks;

- Group IV (protected group): consisted of 20 rats; received first the calculated dose of vitamin B17 orally daily for 5 weeks then colon cancer was induced for the following 5 weeks;

- Group V (treated group): consisted of 20 rats; colon cancer was induced first with DMH injection by the dose mentioned above for 5 weeks, and then received the calculated dose of vitamin B17 for 5 consecutive weeks.

All rats were then sacrificed after 10 weeks.

\section{Clinical assessment}

Initial and final weights were recorded [11]; blood samples were taken for assessment of carcino-embryonic antigen (CEA) levels [12, 26].

\section{Light microscopic study}

A small piece of the distal colon and lumbar vertebrae (cleaned of all soft tissue) were fixed for 24 hours in $10 \%$ neutral buffered formalin then dehydrated in ascending grades of alcohol, cleared and embedded in paraffin. After deparaffinizing, the 3-5 microns thick tissue sections were cut by microtome and were subjected to: haematoxylin and eosin (H\&E) staining for routine histological examination, Masson trichome staining (for colon sections) for detection of collagen fibre deposition, toluidine blue (for bone sections) for assessment of bone mineralisation and immunohistochemical stains for colon sections (Ki-67 [marker of proliferation and detecting the mitotic activity], cytokeratin 20 [CK20 for detecting adenocarcinoma arising from epithelia that normally contain this protein], vascular endothelial growth factor [VEGF for detecting angiogenesis], and Caudal Type Homeobox 2 [CDx2 protein involved in the proliferation and differentiation of intestinal epithelial cells via regulation of intestine-specific gene transcription with a tumour suppressor role]).

\section{Scanning electron microscopic (SEM) study}

Samples from distal colon were subjected to post-fixation in $2 \%$ osmium tetroxide for 2 hours. They were then washed in distilled water for 5 minutes and passed through a series of graded acetones to achieve dehydration. Critical point drying was carried out with liquid carbon dioxide. The specimens were mounted on aluminium slabs using silver conductive paint and gold palladium sputter coating was achieved using a Polaron E5100 sputter coating instrument. The biopsies were examined by a SEM (JEOL JSM35) at an acceleration voltage of $20 \mathrm{kV}$ at electron microscopy unit at Faculty of Medicine, Tanta University [14].

\section{Morphometric study}

Haematoxylin and eosin stain (colon sections): goblet cells number and Mitotic index, H\&E stain (bone sections): number of bone resorption pits, Masson trichome stain: percentage of surface area of collagen fibre deposition, toluidine blue stain: colour intensity and immune-stains: percentage of Ki-67 immuno-positive nuclei, percentage of CK20 
immuno-positive nuclei, area percentage of VEGF and percentage of surface area of $C D \times 2$ positive immune-staining in all groups by using image analyser software (Image J analyser version 1.4308, National Institutes of Health, USA and Digimizer version 4.3.5, MedCalc software). This was done in the Anatomy and Embryology Department, Menoufia University.

\section{Statistical analysis}

Statistical analysis was performed for the initial and final weights, CEA and for the morphometric results. Results were collected, tabulated and statistically analysed by statistical package for social science (SPSS) version 20 on IBM compatible computer. Results were expressed as mean $(\bar{X}) \pm$ standard deviation (SD). Student t-test (a test for significance comparing two groups) was performed [6]. A value of $p<0.05$ was considered significant. Ten rats were used for each [15].

\section{RESULTS}

\section{General results}

Both CC induced group and the treated group showed decreased physical activity when compared to the control group. $20 \%$ and $30 \%$ mortality was recorded for the CC induced group and the treated group, respectively.

\section{Body weight}

Figure 1 shows the body weight changes among the studied groups. Both CC induced group and the treated group showed significant $(p<0.001)$ decrease in their final body weight when compared to their initial ones. The protected group showed a non-significant decrease $(p>0.05)$ in their final weight when compared to their initial one.

\section{Biochemical results}

Figure 2 shows the CEA level among the studied groups. Compared to the control group, animals of the CC induced group showed a statistically significant increase $(p<0.001)$ in their CEA level. The protected group showed a non-significant increase $(p>0.05)$ in its level. The treated one showed a significant increase $(p<0.001)$ in the serum CEA concentration.

\section{Histological results}

All sections of both control and vitamin B17 groups showed nearly similar histological features with no statistically significant differences between them.

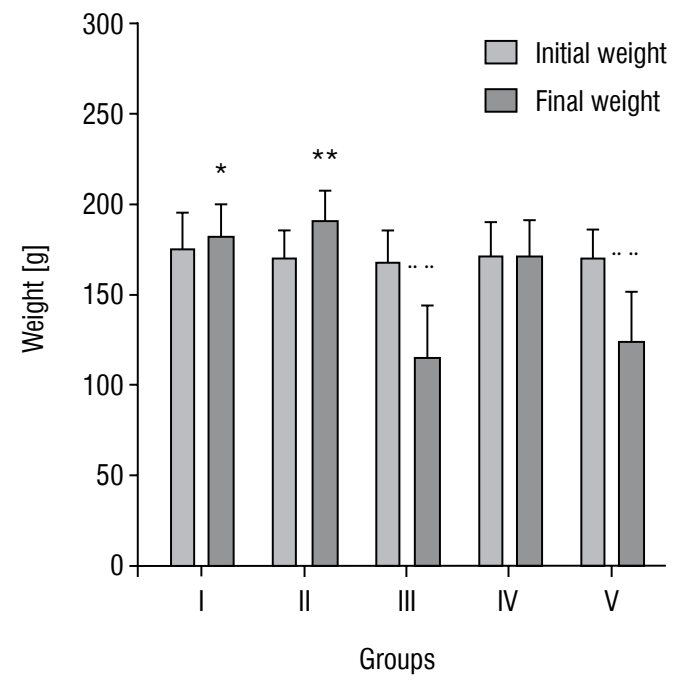

Figure 1. Mean initial and final body weight of all rats (in grams); ${ }^{*},{ }^{* *}$ Significant increase $(p<0.05$ and $p<0.001$, respectively) from the initial body weight; $\bullet$ Significant decrease $(p<0.001)$ from the initial body weight.

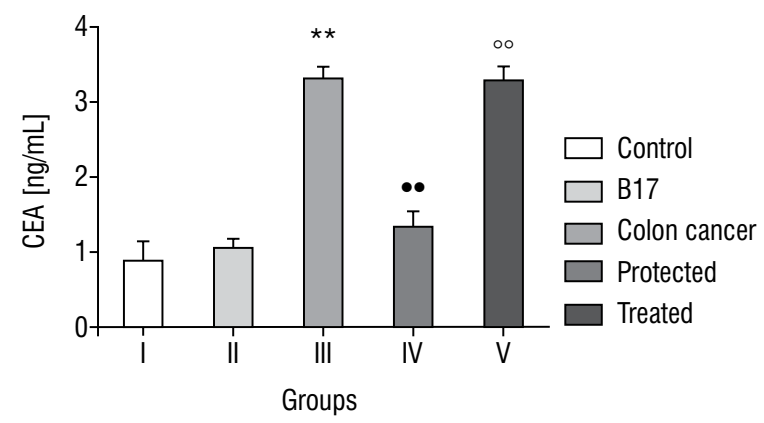

Figure 2. Mean serum carcino-embryonic antigen (CEA) concentration in all groups $(\mathrm{ng} / \mathrm{mL}) ;{ }^{*}$ Significant increase compared with the control group $(p<0.0001) ; \bullet \cdot-$ Significant decrease compared with the colon cancer induced group $(p<0.0001)$; $\circ$ Significant increase compared with the protected group $(p<0.0001)$.

\section{Light microscopic study \\ H\&E stain}

Colon sections. The colonic tissue of control group consisted of: the innermost layer is the mucosa, underneath there is the submucosa, followed by the muscularis propria and finally, the outermost layer - the adventitia. The structure of these layers varies, in different regions of the digestive system, depending on their function. The mucosa showed parallel straight crypts (colonic glands or acini) extending to the muscularis mucosa and separated by the lamina propria. The lining epithelium was composed of principle simple columnar epithelial cells with oval vesicular nuclei and mucous secreting goblet flask-shaped cells with basal dense nuclei. CC induced group (group III) showed disrupted mucosal 
continuity with abnormal sloughing and extrusion of the necrotic debris into the colonic lumen, invasion of the colonic tissue by pleomorphic branching colonic glands of variable shapes and sizes. The abnormal glands were lined with dysplastic elongated hyperchromatic nuclei with an extremely significant $(p<0.0001)$ increase in the number of mitotic figures or stratified multi-layered crowded nuclei with an extremely significant $(p<0.0001)$ reduction of goblet cell number when compared to the control. Group IV showed preservation of the normal colonic architecture of the mucosa which appeared with preserved continuity. Most of the lining epithelial cells appeared with oval vesicular euchromatic nuclei; however, few lining epithelial cells showed mild to moderate dysplasia with elongated hyperchromatic nuclei. Some disruption of the muscularis mucosa with congested blood vessels in the sub-mucosa was evident. This group showed an extremely significant $(p<0.0001)$ decrease in the mitotic index and an extremely significant increase $(p<0.0001)$ in the goblet cell percentage when compared to the CC group. Group V showed more or less distorted colonic architecture with extensive sloughing of the abnormal mucosa into the lumen. The lining epithelial cells showed elongated hyperchromatic nuclei and a non-significant decrease in number of mitotic figures when compared to CC group. An extremely significant $(p<0.0001)$ reduction of goblet cell number was clearly evident. The muscularis mucosa layer was disrupted and the submucosa showed congested dilated blood vessel (Figs. 3-6).

Lumbar vertebra sections. The bone tissue control group showed regular well-organised parallel arrangement of the bone lamellae. The osteocytes within the bone matrix were resident inside their lacunae. The bone marrow space was filled with haematopoietic cells and scattered adipocytes. CC induced group showed an extremely significant increase $(p<0.0001)$ in bone resorption pits in the vicinity of multi-nucleated osteoclasts. The osteocytes appeared with wide lacunae. The bone lamellae lost their parallel well-organised arrangement with areas of sub-periosteal bone necrosis. The bone marrow spaces were widened and invaded by tumour cell nests with signet ring cells. The protected group revealed more or less preserved parallel arrangement of bone lamellae with an extremely significant decrease $(p<0.0001)$ in number of bone resorption pits compared to the CC group, together with presence of several multi-nucleated osteoclasts. The treated group

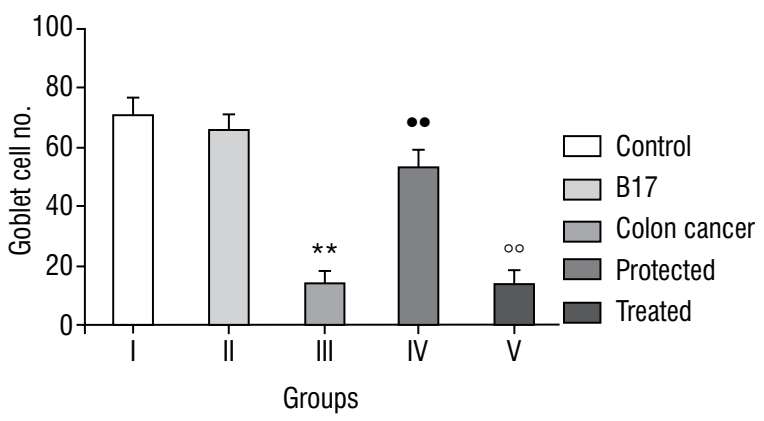

Figure 3. Mean Goblet cells percentage in all groups; ${ }^{*}$ Significant decrease compared with the control group ( $<<0.0001)$; - - Significant increase compared with the colon cancer induced group ( $p<0.0001) ; \circ$ Significant decrease compared with the protected group $(\mathrm{p}<0.0001)$.

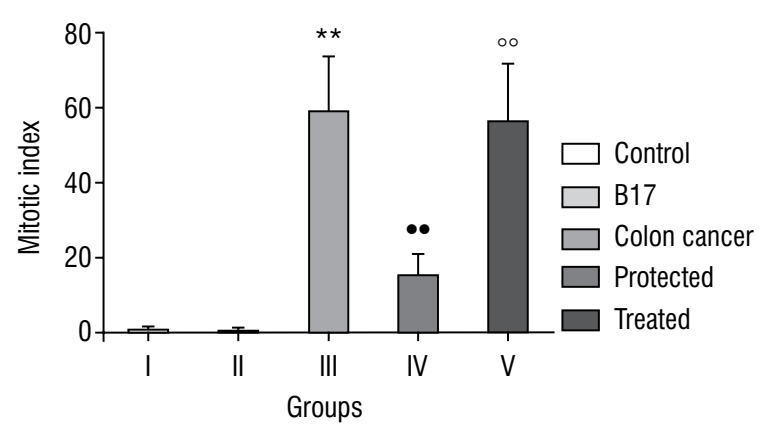

Figure 4. Mean mitotic index in all groups; ${ }^{* *}$ Significant increase compared with the control group $(\mathrm{p}<0.0001)$; $\bullet$ • Significant decrease compared with the colon cancer induced group $(p<0.0001) ; \circ \circ$ Significant increase compared with the protected group $(p<0.0001)$. Mitotic index $=$ Number of mitotic figures $\times 100 /$ Number of all evaluated cells.

showed tumour cell nests invading the bone marrow space and surrounding the destructed trabecular bone that showed a non-significant decrease $(p>0.05)$ of bone resorption pits number compared to CC group, with multi-nucleated osteoclasts (Figs. 7, 8).

\section{Special stains}

Masson trichome stain. When compared with the control group, CC induced group showed an extremely significant increase $(p<0.0001)$ in the surface area percentage of collagen fibre deposition. The protected group showed an extremely significant decrease $(p<0.0001)$ in the same percentage when compared to the CC group. When compared to the CC group, the treated group showed no statistically significant difference $(p>0.05)$ of the same percentage. When compared to the protected group, the treated group showed an extremely significant increase $(p<0.0001)$ in this percentage (Fig. 9). 


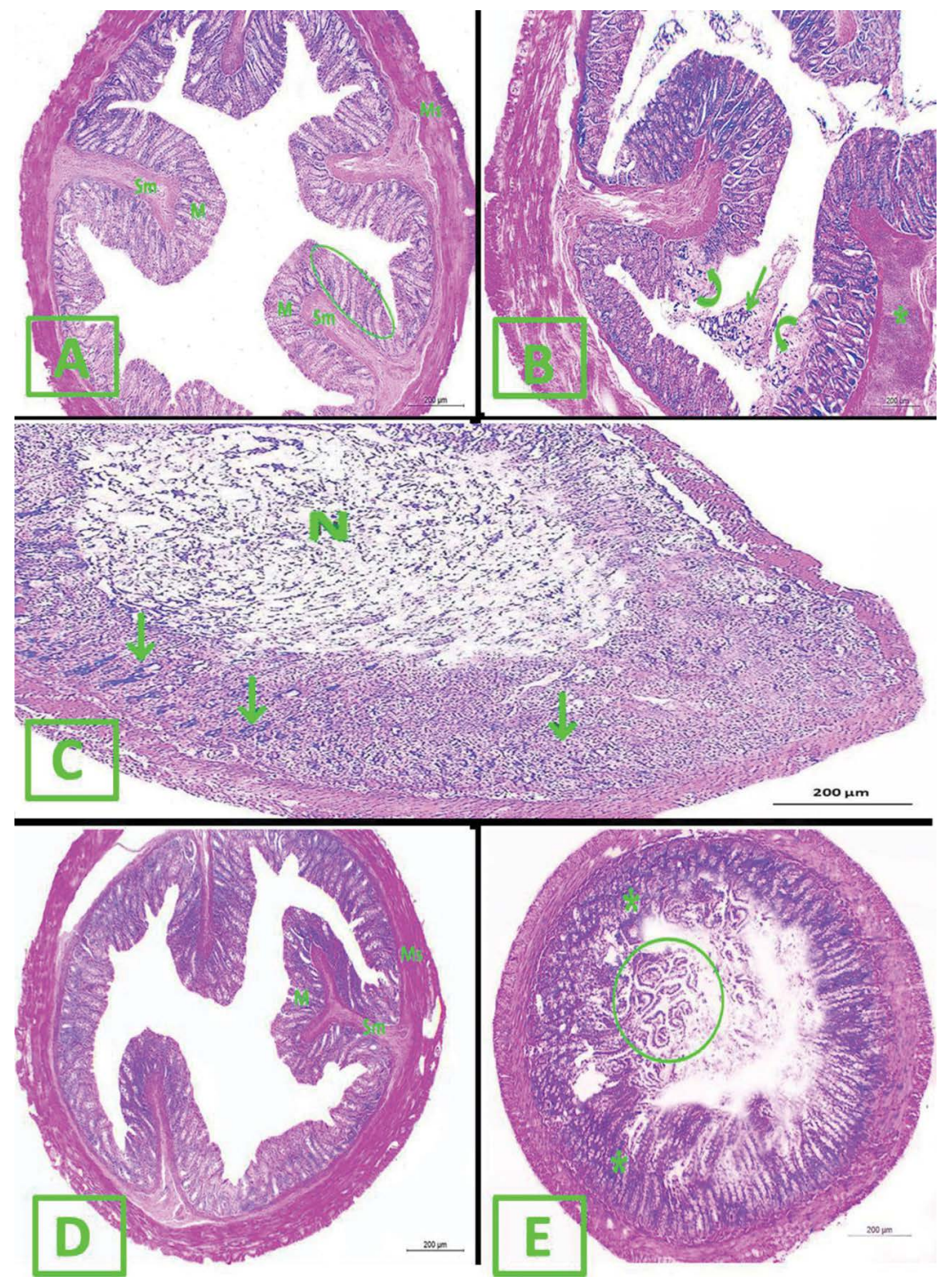

Figure 5. A photomicrograph of colon section in all experimental groups $(\mathrm{H} \& \mathrm{E}$, $\times 40$ ); A. Control group: mucosa (M) with parallel colonic crypts (encircled), submucosa (Sm) and musculosa (Ms); B, C. Colon cancer induced group: disrupted mucosal continuity (curved arrows) with abnormal sloughed tissue extruded into the lumen (thin arrow); C. Severely distorted colonic architecture with complete morphological loss of the mucosa and submucosa (arrows). Necrotic debris filling the colonic lumen (N); D. Protected group: preservation of normal mucosal continuity (M). The submucosa $(\mathrm{Sm})$ and musculosa (Ms) layers appear more or less normal; E. Treated group: more or less distorted architecture. The mucosa and sub-mucosa are infiltrated by irregular crowded colonic glands (asterisks). Sloughing of the crypts into the colonic lumen (encircled).
Toluidine blue stain. When compared with the control group, CC group showed an extremely significant decrease $(p<0.0001)$ in the toluidine blue colour intensity. The protected group showed a very significant increase $(p<0.001)$ in the same percentage when compared to the CC induced group. The treated group showed a non-significant decrease $(p>0.05)$ in toluidine blue colour intensity when compared to the $\mathrm{CC}$ group, while showed an extremely significant decrease $(p<0.0001)$ in toluidine blue colour intensity when compared to the protected group (Fig. 10).

Immuno-stains. Control group showed negative immune-staining for Ki-67, VEGF, mild positive immune-staining for CK20 and marked positive immune-staining for $C D \times 2$. CC group showed strong positive immune-staining for Ki-67, VEGF and CK20, while showed negative immune-staining for $C D \times 2$. The protected group showed mild to moderate positive reactions for all immune-stains. The treated group showed marked positive immune-reaction for Ki-67, VEGF and CK20, while showed negative reaction to CDx2 (Figs. 11, 12).

\section{SEM study}

The control group revealed that the colonic surface mucosa was composed of rounded to oval well-arranged cryptal units. Each unit was covered with concentrically arranged epithelial cells with a central rounded lumen. The crypts were well-delineated by furrows. The cryptal units were loaded with mucous secretion. The CC group showed total loss of the epithelial cells covering the crypts. Moreover, necrotic areas 


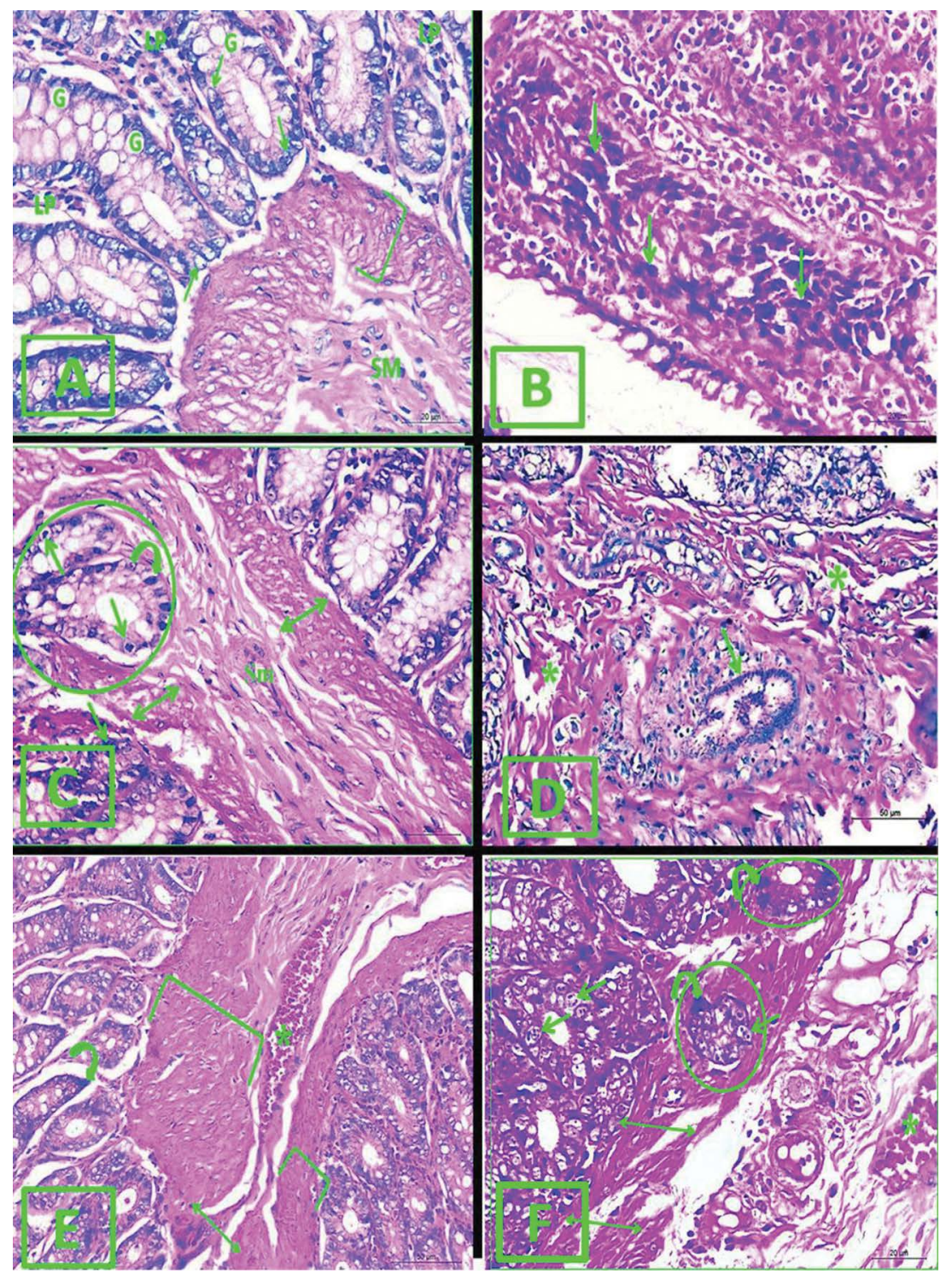

Figure 6. A photomicrograph of colon section in all groups $(H \& E, \times 400)$; A. Control group: normal parallel colonic glands lined by simple columnar epithelial cells with oval vesicular nucleus (arrows) and mucous secreting goblet cells (G) with basal flat nuclei. Lamina propria (LP). Muscularis mucosa (bracket). Submucosa (Sm); B, C, D. Colon cancer group: the atypical crypts lined with epithelial cells with elongated crowded hyperchromatic nuclei (arrows); C. Atypical colonic glands (encircled) invading the muscularis mucosa layer (double headed arrows) reaching the submucosa $(\mathrm{Sm})$. Some nuclei show frequent mitotic figures (thin arrow); D. Abnormal pleomorphic colonic gland (thin arrow) lined with stratified multi-layered hyperchromatic crowded nuclei invading the musculosa layer (asterisks); E. Protected group: the majority of the crypts appear more or less normal. One abnormal shape crypt (curved arrow). Disrupted muscularis mucosa layer (double headed arrow). Asymmetrical appearance of muscularis mucosa (brackets). Congested dilated blood vessel in the submucosa (asterisk); F. Treated group: abnormal glands (encircled) invading a disorganised muscularis mucosa layer (double headed arrow). The lining epithelial cells possess either elongated hyperchromatic nuclei (curved arrows) or frequent mitotic figures (thin arrows). Congested dilated blood vessel in the submucosa (asterisk). of the surface mucosa were clearly evident. The surface mucosa was depleted of mucous secretion. The protected group showed more or less normal crypt units bathed with mucous secretion with preserved both covering epithelium and central lumen. However, some sections showed few abnormal crypts which lost their covering epithelium. The cryptal units of the treated group showed loss of their covering epithelium, others showed obliteration of the central lumen. Necrotic areas of the surface mucosa were clearly evident (Fig. 13).

\section{DISCUSSION}

With the increasing incidence of colon cancer worldwide with its very high morbidity and mortality rates and poor prognosis, several attempts were done to convert it to a preventable disease $[5,11]$. Vitamin B17 (Amygdalin), as a natural substance, may have anti-inflammatory and anti-cancerous effects. The role of vitamin B17 in treating colon cancer is still a matter of controversy. In this study, the elevated serum CEA levels of the colon cancer induced group judged the occurrence of colon cancer which was further supported by the highly significant reduction in the final body weight of this group when compared to the initial one. Furthermore, colon cancer was evidenced by the major pathological lesions affecting the colon; this was in accordance with Moharib et al. [15]. In the current work, the earliest hallmark for colon cancer was the presence of hyperplastic aberrant crypt foci which are abnormal crypts diverging from the normal shape. This was in agreement with Youssef et al. [28]. Hyperplasia of the crypts was further evidenced in this study by the SEM results that showed hyperplastic elevated crypts' epithelial covering. This 


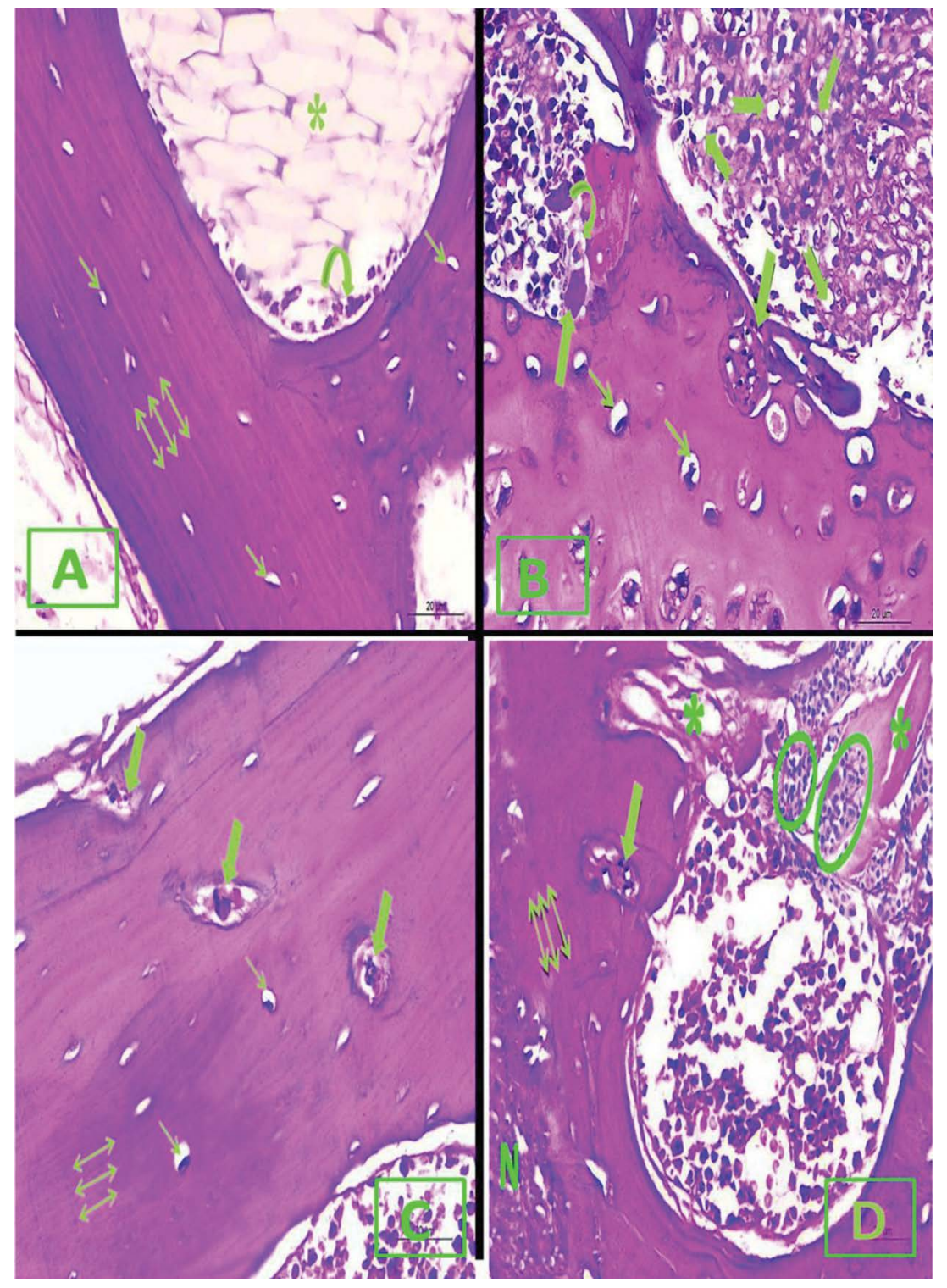

Figure 7. A photomicrograph of lumbar vertebrae section in all groups $(\mathrm{H} \& \mathrm{E}$, $\times 400)$; A. Control group: regular well-organised parallel arrangement of the bone lamallea (double headed arrows). Osteocytes inside their lacunae (thin arrows). The bone marrow spaces can be seen with large vacuolated adipocytes (asterisk) and haematopoietic cells (curved arrow); B. Colon cancer group: multinucleated osteoclasts (thick arrows) at the site of bone scalloping (curved arrow). Osteocytes with wide lacunae (thin arrows). Invasion of the bone marrow spaces by tumour cell nest with signet ring cells (notched arrows); C. Protected group: more or less preserved arrangement of the bone lamellae (double headed arrows). Multi-nucleated osteoclasts (thick arrows). Widening of the osteocytes' lacunae (thin arrows); D. Treated group: distorted arrangement of the bone lamellae (double headed arrows). Tumour cell nests (encircled) invading destructed bone trabeculae (asterisks). Multi-nucleated osteoclast (thick arrows). Area of bone necrosis (N).

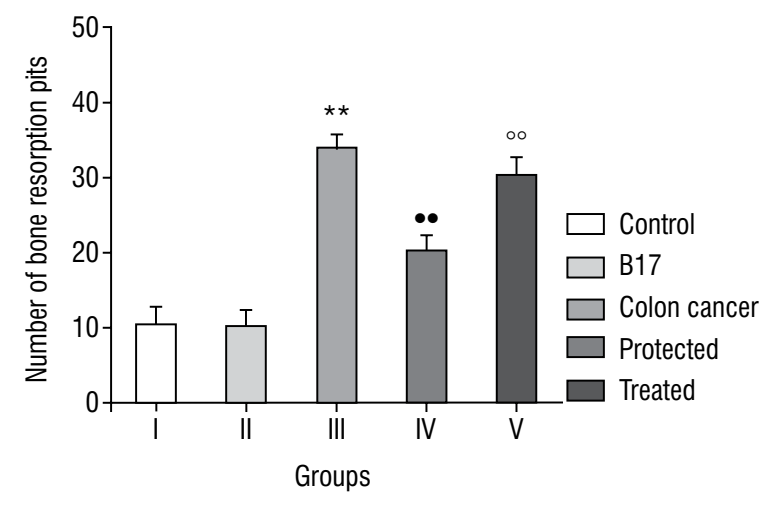

Figure 8. Mean number of bone resorption pits; ${ }^{*}$ Significant increase compared with the control group $(p<0.0001)$;

- Significant decrease compared with the colon cancer induced group ( $<<0.0001)$; $\circ$ Significant increase compared with the protected group $(p<0.0001)$. was supported by Paulsen et al. [20]. Sanganna and Kulkarni [21] stated that hyperplasia might be due to the oxidative stress involved in the process of tumour development. This mechanism might explain the highly significant increase in the mitotic index of this group when compared to control as shown in our results. Furthermore, in the present work, the CC induced group showed intense positive immunoreaction for Ki-67 which might prove the enhanced proliferative activity. All degrees of dysplasia have been reached; mild, moderate and severe dysplasia with finally the anaplasia or the carcinoma stage. This was in agreement with Jucaa et al. [11] who used the same methodology for completion of experimental carcinogenesis by $\mathrm{DMH}$. Colon cancer group in this 


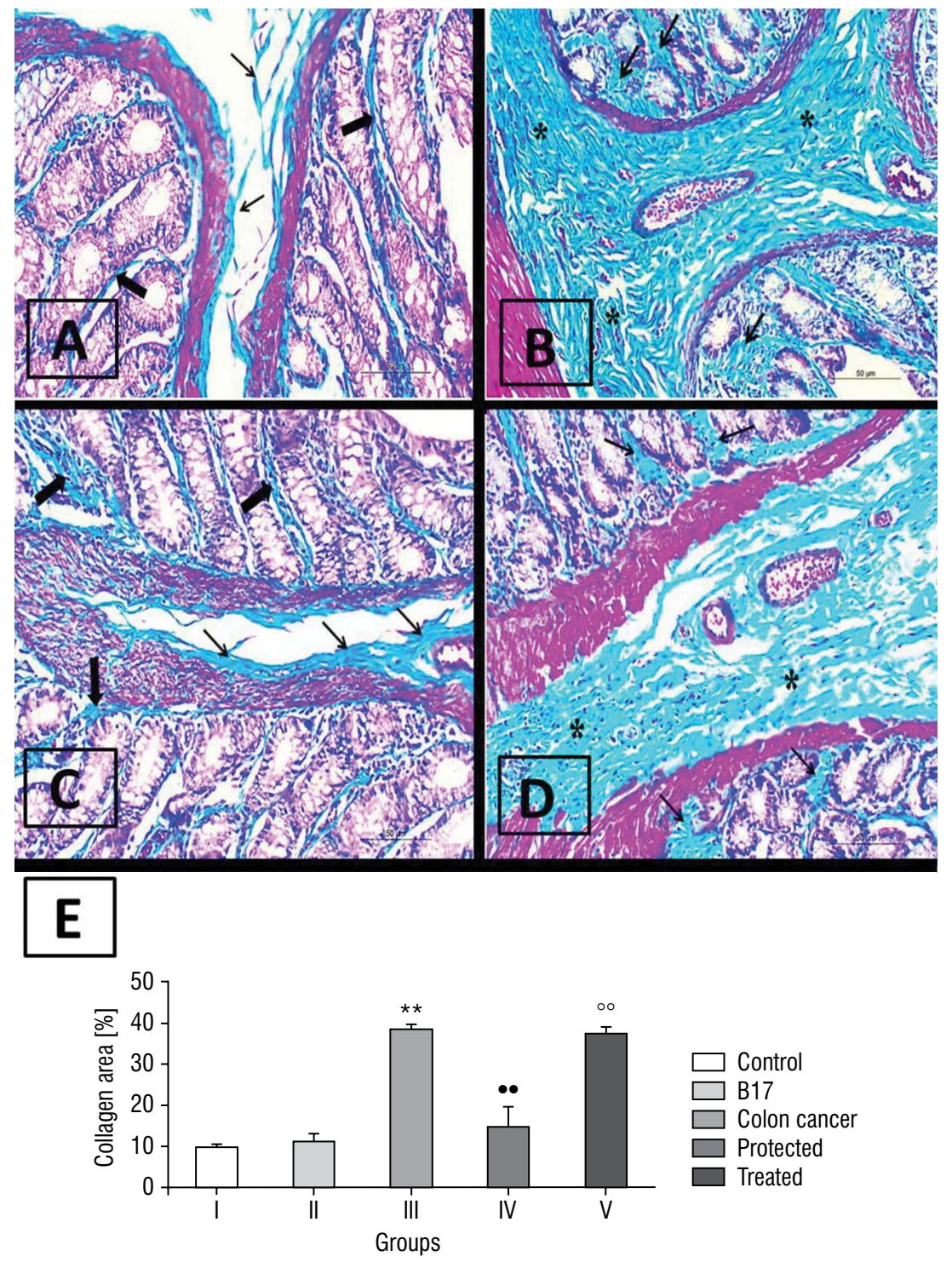

Figure 9. Photomicrographs of Masson trichome stained colon sections of all groups ( $\times 400)$; A. Control group: minimal amount of collagen fibre deposition (arrows); B. Colon cancer induced group: large amount of collagen fibre deposition (arrows and asterisks); C. Protected group: small amount of collagen deposition (thick and thin arrows); D. Treated group: large amount of collagen fibre deposition (arrows and asterisks); E. Foote notes: ${ }^{*}$ Significant increase compared with the control group $(\mathrm{p}<0.0001) ; \bullet \cdot$ Significant decrease compared with the CC group $(p<0.0001) ; \circ$ o Significant increase compared with the protected group $(p<0.0001)$. study showed strong positive immune-reaction for CK20; which emphasized reaching the adenocarcinoma stage; this was in accordance with Nabil et al. [16]. In the present study, CC induced group showed a highly significant decrease in the number of Goblet cells or their complete absence. This was further evidenced by our SEM study which revealed absence of the mucous secretion in this group. In this study, CC group showed a highly significant increase in the percentage of collagen fibre deposition. Fibrosis might suggest the presence of chronic inflammation, thus chronic exposure of fibroblasts to inflammatory mediators may drive their transition to activated myofibroblasts with consequent abnormal collagen production. Colon cancer induced group showed highly significant decrease in the percentage of CDx2 positively stained surface area; this was in agree- ment with Sangeetha and Nalini [22]. In the current study, angiogenesis (formation of new capillaries from pre-existing blood vessels) as one of the most leading factors to develop bone metastasis has been proved as CC group showed strong positive expression of VEGF. Lumbar spine was the site of choice to assess the possibility of bone metastasis. This was in accordance with Jimi et al. [10], who postulated that the lumbar spine, followed by the pelvis were the most common sites of bone metastasis in colon cancer (62.16-55.40\%, respectively). Cassar et al. [3] attributed vertebral metastasis from colon cancer to a vertebral venous plexus named Baston's plexus which was considered to be the main source of spreading. In the present study, bone metastasis was evident due to presence of multiple osteolytic lesions and resorption pits. Increased activity of the 


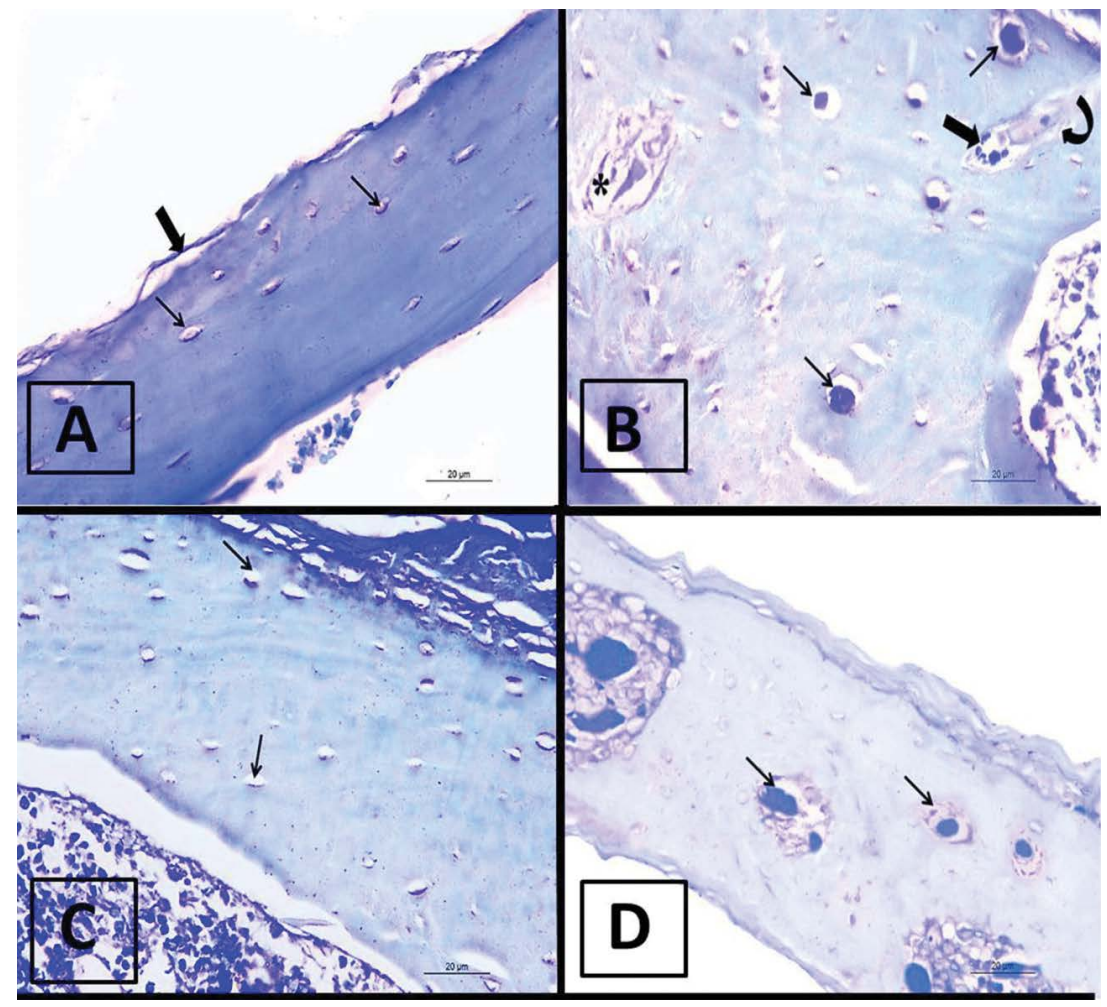

E

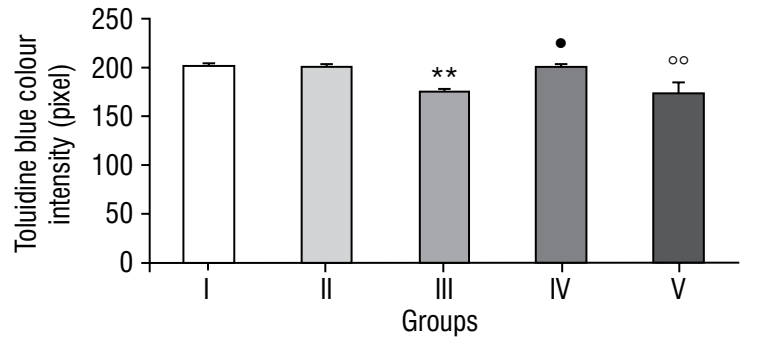

osteoclasts revealed the presence of osteolytic type of bone metastasis. This was in accordance with Macedo et al. [13]. The presence of tumour cell nests inside the bone marrow cavity emphasized the metastasis. These results were in line with Vatandoust et al. [27] who reported that signet ring cell stage of colon cancer had a high rate of bone osteolytic metastasis. In the current work, when compared to the CC group, the protected group showed nearly normal physical activity, average food intake, a non-significant difference between their final body weight and their initial one. No deaths occurred among this group, marvellous regenerative changes proved by the significant improvement in serum CEA level and the impressive improvement of the disrupted histology that appeared very close to reach the normal appearance.
Figure 10. Photomicrographs of toluidine blue stained lumbar vertebral sections of all groups $(\times 400)$; A. Control group: dark blue staining of the bone sections; B. Colon cancer group: faint blue staining of the bone sections; C. Protected group: average blue staining of the bone sections; D. Treated group: pale blue staining of the bone sections; E. Foote notes: ${ }^{* *}$ Significant decrease compared with the control group $(p<0.0001)$; $\bullet$ Significant increase compared with the colon cancer induced group $(p<0.05)$; $\circ$ Significant decrease compared with the protected group $(p<0.0001)$.
On the contrary, the treated group did not show any impressive improvement compared with CC induced and protected groups. The present study showed significant decrease in collagen fibre deposition of the protected group when compared to the treated one. This might suggest the anti-fibrotic action of vitamin B17. This could be explained as vitamin B17 caused regression of inflammation with subsequent regressed fibrosis. This was in accordance with Bottinger and Bitzer [1] who reported that vitamin B17 might enhance serum butyrate concentration which caused suppression of pro-fibrotic cytokine transforming growth factor beta 1 (TGF- $\beta 1$ ). Moreover, Guo et al. [8] studied the effect of amygdalin in suppressing kidney fibroblast proliferation and TGF- $\beta 1$ secretion in the lymphocytes and thus was able to 


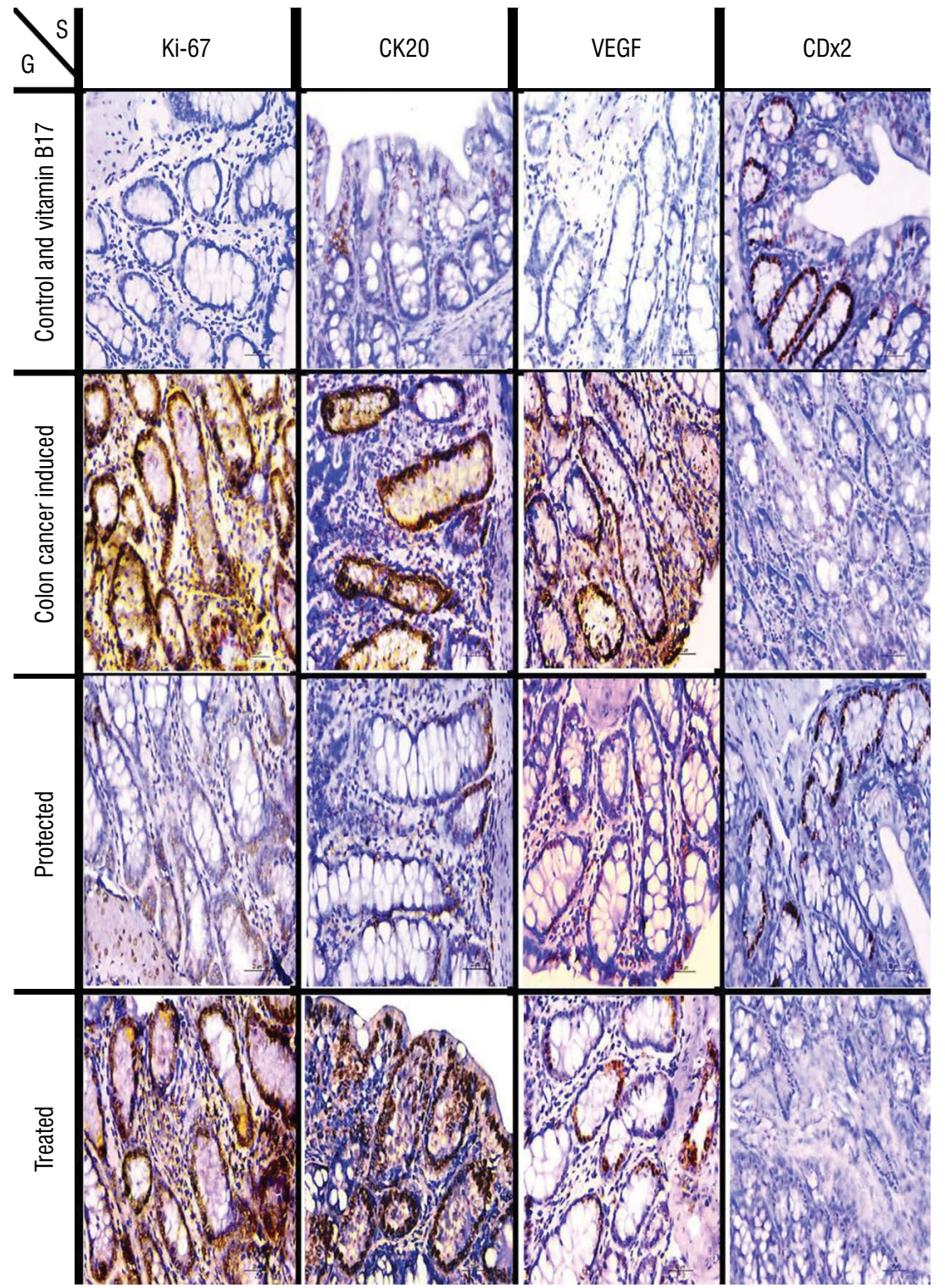

Figure 11. Photomicrographs of immuno-stained colon sections of all groups $(\times 400)$; Control and vitamin B17 groups: negative immune-staining for Ki-67, CK20, VEGF and positive for CDx2. CC induced group: strong positive immune-staining for Ki-67, CK20, VEGF and negative for $\mathrm{CDx}$; Protected group: mild to moderate immune-reaction for all immune-stains; Treated group: strong positive immune-staining for Ki-67, CK20 and VEGF, but negative reaction to $C D \times 2$ immuno-stain; S - stain; G - group; rest abbreviations - see text.

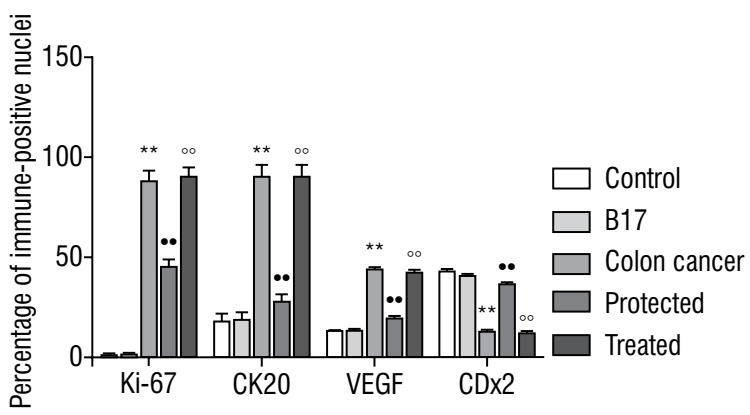

Figure 12. Mean area percentage of immune-positive nuclei (\%) in all groups: **Significant increase $(p<0.0001)$ compared with the control group (for Ki-67, CK20 and VEGF) and significant decrease $(p<0.0001)$ compared with the control group for CDx2; - - Significant decrease compared with the colon cancer group for Ki-67, CK20 and VEGF and significant increase compared with colon cancer group for CDX2 ( $p<0.0001)$; $\circ$ Significant increase from compared with the protected group for Ki-67, CK20 and VEGF and significant decrease from protected group for CDx2 $(p<0.0001)$; abbreviations - see text. significantly postpone the process of renal interstitial fibrosis, which further proved the anti-fibrotic effect of Amygdalin. Furthermore, they hypothesized that the mechanisms which might be involved include increasing the secretion of type I collagenase, inhibiting kidney fibroblast proliferation, accelerating apoptosis and suppressing type I collagen synthesis. In the present study, the protected group showed mild positive immunoreaction for $\mathrm{Ki}-67$ which might prove the inhibition of proliferative activity. This finding was in agreement with Park et al. [19]. The mechanisms of vitamin B17 in defeating cancer cells were uncertain; however, Cassiem and De-Kock [4] performed a very recent study on vitamin B17's anti-cancer role. Being selective for destructing cancer cells which lack rhodanese enzyme, vitamin B17 exerts its anti-oxidant 

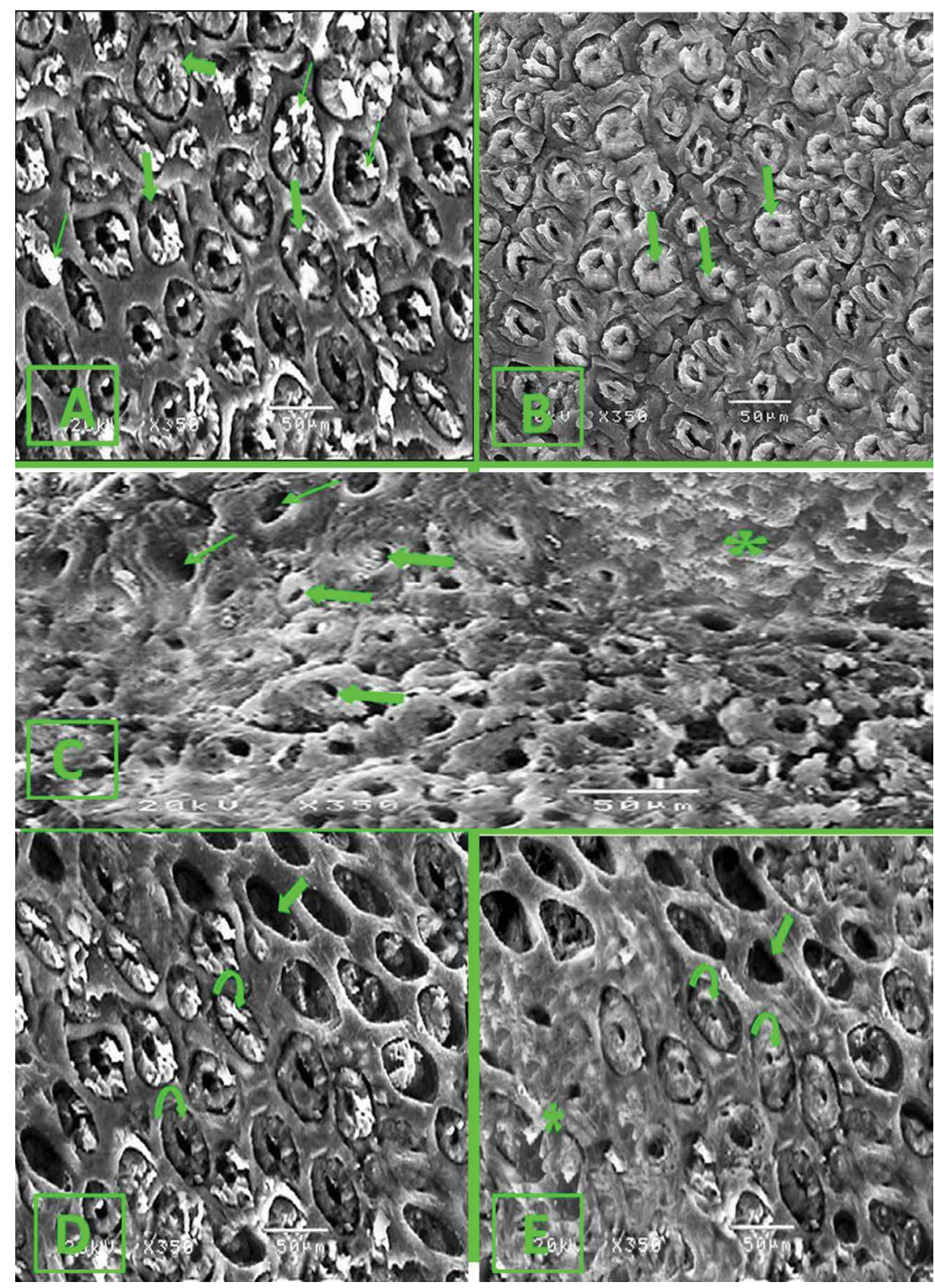

Figure 13. Scanning electron micrographs of colon sections of all groups (SEM, $\times 350$ ); A. Control group: rounded to oval crypt units (encircled) covered with concentrically arranged epithelial cells, each unit is demarcated by a furrow (thick arrow) with a lumen at the centre (asterisk). Mucous secretion can be noticed (thin arrows); B. Colon cancer induced group: elevated crypt units with hyperplasia (arrows); C. Colon cancer group: crypts with narrowed slit like lumen (thick arrows); others show wide crypt lumen with total loss of the cells covering the crypts (thin arrows).

A necrotic area can be observed (asterisk); D. Protected group: some crypts appear more or less normal (curved arrows); others show complete loss of their covering cells (thick arrow); E. Treated group: crypts with obliterated lumen (curved arrows); others show total loss of their covering epithelium (thick arrow). A large necrotic area can be noticed (asterisk). action. Normal cells do have the rhodanese enzyme which neutralizes vitamin B17 converting it to a harmless compound; thiocyanate. Meanwhile, cancer cells lack rhodanese enzyme, so cyanide radical is liberated. Moreover, Song and Xu [24] added that Amygdalin could significantly increase polyhydroxyalkanoates that induced human peripheral blood T lymphocyte proliferation with secretion of interleukin 2 and interferon gamma, and then inhibiting the secretion of TGF- $\beta 1$, therefore enhancing the immune function. In the current work, treatment of colon cancer with vitamin B17 did not either induce improvement or reversal of the pathological changes that occurred. This might be due to the progressive and irreversible uncontrolled progressive cancer cell division and bone metastasis. Ki-67, CK20 and VEGF all showed intense positive immunostaining when compared to the control group.

\section{CONCLUSIONS}

Our findings revealed that daily intake of vitamin B17 may play an important role as a chemo-preventive, anti-cancer, anti-inflammatory, anti-fibrotic and anti-oxidant agent. Furthermore, this study proved the uselessness of vitamin B17 in curing colon cancer.

\section{Acknowledgements}

Acknowledgment to all members of Human Anatomy and Embryology Department Faculty of Medicine, Menoufia University, Egypt. 


\section{REFERENCES}

1. Bottinger EP., Bitzer M. TGF-beta signaling in renal disease. J Am Soc Nephroll. 2002; 13(10): 2600-2610, doi: 10.1097/01.asn.0000033611.79556.ae.

2. Carethers JM. Clinical and genetic factors to inform reducing colorectal cancer disparitites in African Americans. Front Oncol. 2018; 8: 531, doi: 10.3389/fonc.2018.00531, indexed in Pubmed: 30524961.

3. Cassar N, Cresswell AB, Moran B. Oligometastatic colorectal cancer: is single-site bony colorectal metastasis a treatable condition? Int J Colorectal Dis. 2017; 32(8): 1229-1231, doi: 10.1007/s00384-017-2780-1, indexed in Pubmed: 28213624

4. Cassiem W, de Kock M. The anti-proliferative effect of apricot and peach kernel extracts on human colon cancer cells in vitro. BMC Complement Altern Med. 2019; 19(1): 32, doi: 10.1186/s12906-019-2437-4, indexed in Pubmed: 30696432.

5. Chari KY, Polu PR, Shenoy RR. An appraisal of pumpkin seed extract in 1, 2-dimethylhydrazine induced colon cancer in Wistar rats. J Toxicol. 2018; 2018: 6086490, doi: 10.1155/2018/6086490, indexed in Pubmed: 30245714.

6. De Muth JE. Presentation Models in Basic Statistics and Pharmaceutical Statistical Applications, 3rd edition. CRC Press. 2014; 4: 95, doi: 10.1201/b16842.

7. De-Souza AS, Costa-Casagrande TA. Animal models for colorectal cancer. Arq Bras Cir Dig. 2018; 31(2): e1369, doi: 10.1590/0102-672020180001e1369, indexed in Pubmed: 29972397.

8. Guo J, Wu W, Sheng M, et al. Amygdalin inhibits renal fibrosis in chronic kidney disease. Mol Med Rep. 2013; 7(5): 1453-1457, doi: 10.3892/mmr.2013.1391, indexed in Pubmed: 23525378.

9. Jaswal V, Palanivelu J, C R. Effects of the Gut microbiota on Amygdalin and its use as an anti-cancer therapy: Substantial review on the key components involved in altering dose efficacy and toxicity. Biochem Biophys Rep. 2018; 14: 125-132, doi: 10.1016/j.bbrep.2018.04.008, indexed in Pubmed: 29872744.

10. Jimi Si, Yasui T, Hotokezaka M, et al. Clinical features and prognostic factors of bone metastases from colorectal cancer. Surg Today. 2013; 43(7): 751-756, doi: 10.1007/ s00595-012-0450-z, indexed in Pubmed: 23224335.

11. Jucaa $M$, Bandeira $B$, Carvalho $D$, et al. Comparative study of 1,2-dimethylhydrazine and azoxymethane on the induction of colorectal cancer in rats. J Coloproctol. 2014; 34(3): 167-173, doi: 10.1016/j.jcol.2014.06.003.

12. Li AA, Yuan ZC, Jia-Ming $L$, et al. The risk factors for bone metastases in patients with colorectal cancer. Medicine. 2018; 97(40): e12694.

13. Macedo F, Ladeira $K$, Pinho F, et al. Bone metastases: an overview. Oncol Rev. 2017; 11(1): 321, doi: 10.4081/oncol.2017.321, indexed in Pubmed: 28584570.

14. Metwally IH, Shetiwy M, Elalfy AF, et al. Epidemiology and survival of colon cancer among Egyptians: a retrospective study. J Coloproctol (Rio J). 2018; 38(1): 24-29.

15. Moharib SA, Abd El Maksoud N, Ragab HM, et al. Anticancer activities of mushroom polysaccharides on chemically-induced colorectal cancer in rats. J Applied Pharmaceutical Sci. 2014; 4(7): 54-63, doi: 10.7324/japs.2014.40710.
16. Nabil HM, Hassan BN, Tohamy AA, et al. Radioprotection of 1,2-dimethylhydrazine-initiated colon cancer in rats using low-dose $\gamma$ rays by modulating multidrug resistance-1, cytokeratin 20, and $\beta$-catenin expression. Hum Exp Toxicol. 2016; 35(3): 282-292, doi: 10.1177/0960327115584687, indexed in Pubmed: 25926526.

17. Nasir NL, Kamsani NE, Mohtarrudin N, et al. Anticarcinogenic activity of Muntingia calabura leaves methanol extract against the azoxymethane-induced colon cancer in rats involved modulation of the colonic antioxidant system partly by flavonoids. Pharm Biol. 2017; 55(1): 2102-2109, doi: 10.1080/13880209.2017.1371769, indexed in Pubmed: 28872373.

18. Nour A, Azar B, Rabata A. The effect of amygdalin in the treatment of squamous cell carcinoma induced in the buccal pouch of golden syrian hamster. J Dental Med Sci. 2016; 15(2): 75-79.

19. Park HJ, Yoon SH, Han LS, et al. Amygdalin inhibits genes related to cell cycle in SNU-C4 human colon cancer cells. World J Gastroenterol. 2005; 11(33): 5156-5161.

20. Paulsen JE, Namork E, Alexander J. Scanning electron microscopy of colonic lesions in 1,2-dimethylhydrazine-treated rats. Anticancer Res. 2005; 25(6B): 3883-3888, indexed in Pubmed: 16309175.

21. Sanganna B, Kulkarni AR. Effect of citrus reticulata essential oil on aberrant crypt foci (acf) development in 1,2-dimetylhydrazine induced colon carcinogenesis rats. Int J Pharmac App. 2013; 4(1): 29-37.

22. Sangeetha N, Nalini N. Silibinin modulates caudal-type homeobox transcription factor (CDX2), an intestine specific tumor suppressor to abrogate colon cancer in experimental rats. Hum Exp Toxicol. 2015; 34(1): 56-64, doi: 10.1177/0960327114530741, indexed in Pubmed: 24740923.

23. Shewatatek G, Wajana LL, Getinet Y. Coffee arabica complies chemo-preventive activity against $\mathrm{DMH}$ induced colorectal cancer in experimental rat model. J Med Diagn Meth. 2017; 6: 1-3.

24. Song Z, Xu X. Advanced research on anti-tumor effects of amygdalin. J Cancer Res Ther. 2014; 10 Suppl 1: 3-7, doi: 10.4103/0973-1482.139743, indexed in Pubmed: 25207888

25. Usher-Smith J, Walter F, Emery J, et al. Risk prediction models for colorectal cancer: a systematic review. Cancer Prev Res. 2015; 9(1): 13-26, doi: 10.1158/1940-6207. capr-15-0274.

26. Van Herck H, Baumans V, Brandt CJ, et al. Blood sampling from the retro-orbital plexus, the saphenous vein and the tail vein in rats: comparative effects on selected behavioural and blood variables. Lab Anim. 2015; 35(2): 131-139, doi: 10.1258/0023677011911499, indexed in Pubmed: 11315161.

27. Vatandoust S, Price TJ, Karapetis CS. Colorectal cancer: Metastases to a single organ. World J Gastroenterol. 2015; 21(41): 11767-11776, doi: 10.3748/wjg.v21.i41.11767, indexed in Pubmed: 26557001.

28. Youssef KM, Ezzo AM, El-Sayed MI, et al. Chemopreventive effects of curcumin analogs in DMH-Induced colon cancer in albino rats model. Future J Pharmac Sci. 2015; 1: 57-72. 